\title{
FLAT TYPE THICK FILM INDUCTIVE SENSORS
}

\author{
D. MARIOLI, E. SARDINI* and A. TARONI \\ Dipartimento di Elettronica per l'automazione and I.N.F.M., Facoltà di Ingegneria, \\ Università di Brescia, Via Branze 38, 25123 Brescia, Italy
}

(Received 30 May 2002; In final form 3 July 2002)

\begin{abstract}
Two thick film flat-type inductive sensors are described and tested for distance and profile measurement. The first one is a single-layer spiral while the second one is a multi-layer structure consisting of ten spirals one over the other. The paper describes their geometric configurations together with their simulated magnetic fields and it reports the results from the characterization test i.e. the series-equivalent circuit parameters, the sensitivity and the cross-sensitivity to temperature. An experimental analysis of the sensitivity suggests that optimized values are obtained by an appropriate choice of the working frequency. The sensors are shielded against e.m. noise coming from the nonsensitive area. Moreover, two sensors have been tested in the laboratory using the single layer as a distance sensor and the multi-layer as a transducer for the measurement of a metallic object profile. The results of the tests show a maximum sensitivity of $14 \mathrm{mV} / \mu \mathrm{m}$ and a resolution of $0.6 \mu \mathrm{m}$ for the single layer, while the multi layer one reconstructs the profile with an axial resolution of a few microns and a lateral resolution better than $200 \mu \mathrm{m}$.
\end{abstract}

Keywords: Please supply

\section{INTRODUCTION}

In the field of proximity sensors, inductive sensors are commonly used because of their robustness to environmental conditions [1] and their low costs. Inductive sensors obtained over a planar surface such as a printed circuit board or a silicon chip have been reported in the literature [2-5] recently. They are proposed for different applications such as coin recognition [2], measurements of metallic object profile [2,5] and for hole detection in a step-motor controlling operation [4]. These sensors have good metrological performances, especially high axial resolution even if the lateral resolution is lower. The axial and lateral are defined as the directions along the perpendicular or parallel axis with respect to the surface of the sensor.

In all cases, the physical effects exploited are (i) the eddy current in target objects, mainly relevant on non-ferromagnetic materials and (ii) the reluctance change of the magnetic circuit, mainly relevant with target object of ferromagnetic material. Conditioning electronics based on a differential relaxation oscillator offers good metrological characteristics combined with low cost [6-8], but the output depends on the two physical effects in an opposite relation. For example different conditioning electronics based on a synchronous demodulation

* Corresponding author. Tel.: +39-0303517800; Fax: +39-030380014; E-mail: sardini@ing.unibs.it 
technique, can extract two signals: each one depends more directly and separately on the two physical effects with a lower cross sensitivity to temperature.

A flat inductive sensor can be, also, integrated together with its conditioning electronics over a silicon substrate [9]. However, micro-machining techniques require large production (more than $10^{5}-10^{6}$ ) in order to achieve competitive cost-performance ratio, while thick film technology becomes a strategic factor in the case of production volume with diversified models, due to little investment and designing cost. Moreover, as in the case of the silicon inductive sensor, the signal conditioning circuits can be manufactured over the sensor substrate providing low-cost units.

In the paper, two different types of inductive sensors obtained by thick film technology are presented. The first one is a single layer planar whose shape is a spiral of 16 turns, screen printed over an alumina substrate; the second one is obtained by overlapping spirals one over the other packed in a sandwich structure. Simulations of the magnetic field generated by the single layer with different geometric characteristics have been compared and a guideline in the design of the spiral is proposed. The dependence of the sensitivity on the two physical effects has been analyzed and evaluated, showing that the optimized value depends on the choice of frequency. The sensors, together with their conditioning electronics have been tested in the laboratory proving that the single layer can be used as a distance sensor while the multi-layer should be used in profile measurement applications.

\section{SENSORS DESCRIPTION}

\subsection{The Single Layer Sensor}

Figure 1(a) shows the shape of the sensor schematically; it consists of a spiral track of conductive material ( $\mathrm{PdAg}$ conductive paste $\mathrm{C} 1075$ Heraeus) printed on an alumina substrate whose dimensions are $50 \times 25 \mathrm{~mm}$ with a thickness of $0.5 \mathrm{~mm}$. The external diameter is $18 \mathrm{~mm}$, the width and the thickness of the tracks are 250 and $20 \mu \mathrm{m}$ respectively, while the pitch is $500 \mu \mathrm{m}$. An isolating layer (IP 9117 Heraeus), $20 \mu \mathrm{m}$ thick covers the entire spiral. The equivalent series circuit, consisting of a resistor $\left(R_{S}\right)$ and an inductor $\left(L_{s}\right)$, is measured by the HP 4194A impedance analyzer, without the presence of any objects close to the sensor, giving $R_{s}=4.4 \Omega$ and $L_{s}=2 \mu \mathrm{H}$ at $150 \mathrm{kHz}$ frequency. The parasitic capacitance $C_{p}$, parallel to the series of $R_{S}$ and $L_{s}$, is negligible at this frequency, being the impedance phase close to $\pi / 2$ from few $\mathrm{MHz}$ up to $40 \mathrm{MHz}$.

The 3D magnetic field generated by the sensor is simulated by the Maxwell program and the results are compared with those obtained by a sensor with a shape shown in Figure 1(b). The two spirals are equal but the one reported in Figure 1(b) has the internal terminal starting away from its center. With reference to the coordinate system of the same Figure 1, the magnetic flux density $B$ is simulated over three lines defined for $0<x<12, y=0$ and $z=d$ where $d$ is equal to 100,300 and $500 \mu \mathrm{m}$. Figure 2 reports on the simulation results: in (a) the curves corresponding to the spiral of Figure 1(a) while in (b) those obtained from spiral in Figure 1(b). The curves in Figure 2(a) are more uniform and the greatest values are concentrated in the center of the spiral. Moreover, looking at the curves for $d=300 \mu \mathrm{m}$ in both graphes, the $B$ magnitude decreases to $50 \%$ of its maximum at about $x=3$ and $x=7 \mathrm{~mm}$ meaning about $35 \%$ and $60 \%$ of its external diameter, for the shape of Figure 1(a) and 1(b) respectively: a more concentrated magnetic field is obtained with the shape of Figure 1(a).

To shield the sensor from interferences outside of the sensitive area, a ferromagnetic film has been screen printed on the opposite face of the alumina substrate thus increasing the 

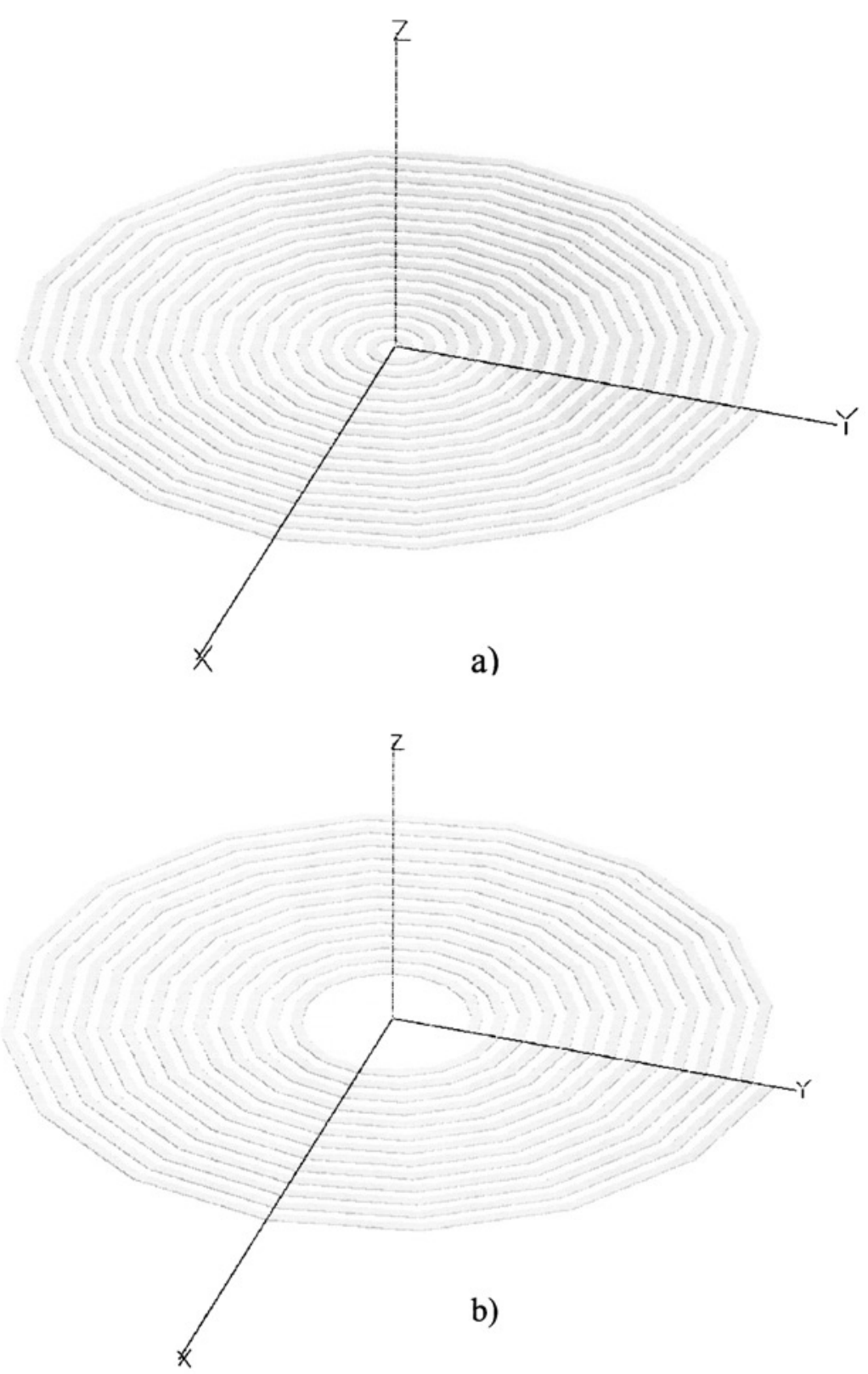

FIGURE 1 Schematic drawings of single layer planar sensor in (a), while in (b) the same sensor but with a hole in the center.

inductance value as well as the sensitivity. Because a ferromagnetic powder was not commercially available, we tried to obtain it by grinding a commercial ferrite and adding the insulating paste IP9117 in the measure of $25 \%$ of the total volume. The thickness of this layer has been increased in various steps from 0.1 up to $1 \mathrm{~mm}$, the maximum possible height, reducing the reluctance of the magnetic path and consequently increasing the $L_{s}$ values from 2.06 up to $2.40 \mu \mathrm{H}$ at $150 \mathrm{kHz}$ frequency. Anyway, higher values of $L_{s}$ and $R_{s}(3.01 \mu \mathrm{H}$ and $4.38 \Omega$ at $150 \mathrm{kHz}$ ) have been reached by attaching a $25 \mu \mathrm{m}$ layer of mu-metal to the back of the 


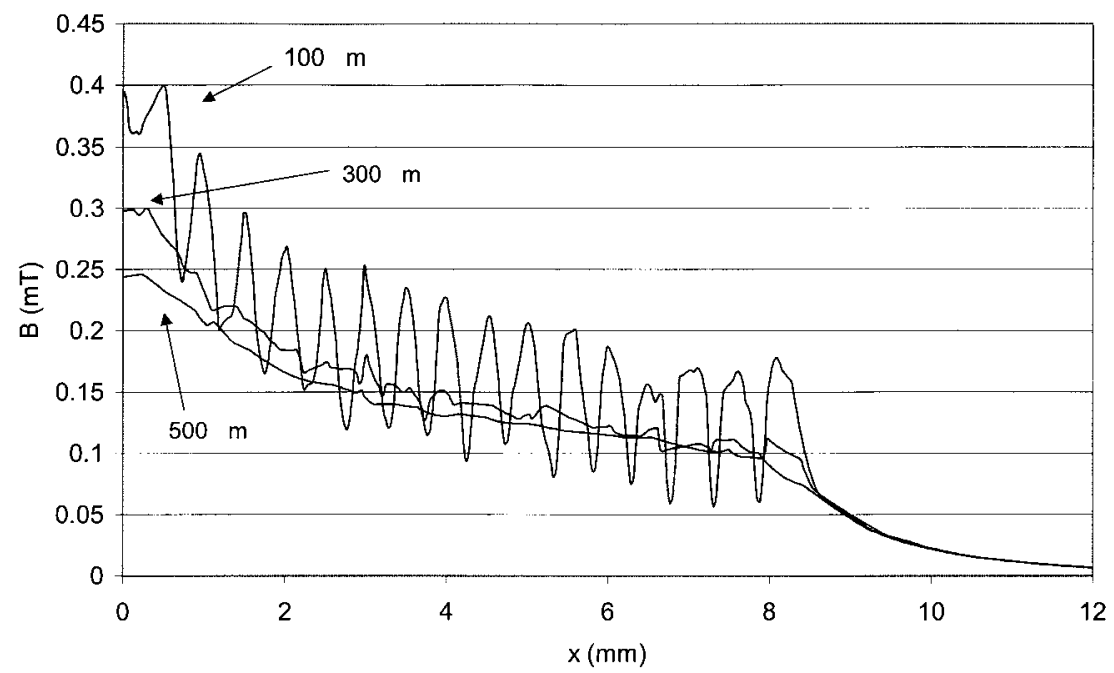

(a)

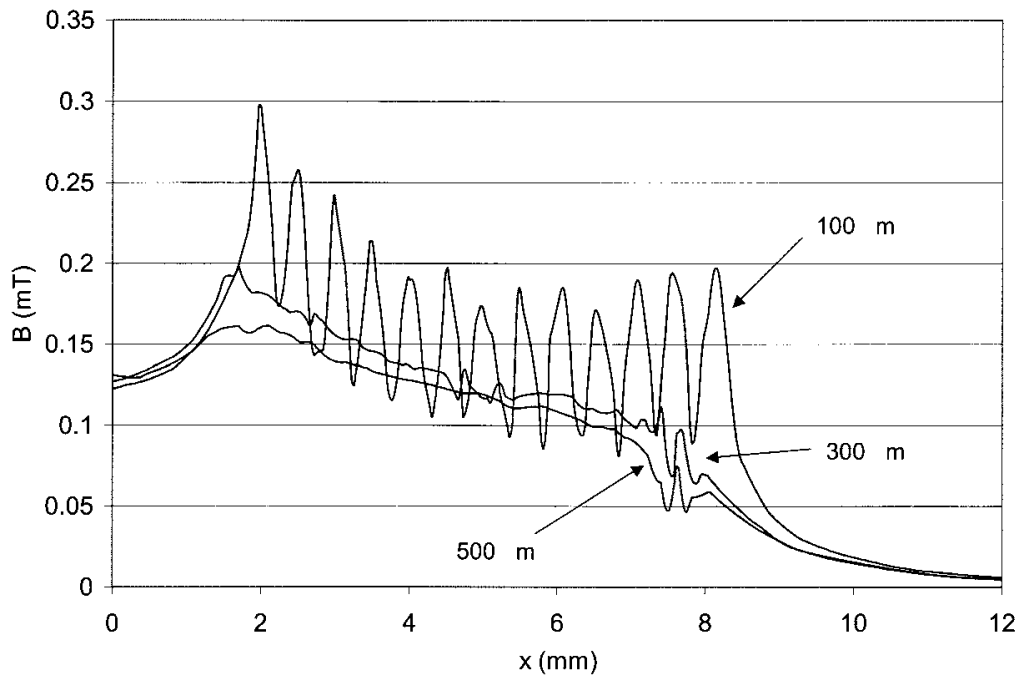

(b)

FIGURE 2 Magnitude of the magnetic flux density $B$ as a function of $x$ along lines defined as $y=0$ and $z=d$ with $d$ reported as parameter. In (a) the magnitude of the spiral of Figure 1(a) and in (b) that of Figure 1(b).

substrate. Figure 3 reports the $L_{s}$ (continuous line) and $R_{S}$ (dot lines) values as a function of frequency with the different solutions adopted. Moreover, the figure shows that the mu-metal layer is useful in a limited frequency range because, at a very high frequency, the effect of eddy-current losses is dominant. $C_{p}$ is again negligible at $150 \mathrm{kHz}$ since the impedance phase rises up to $4 \mathrm{MHz}$ when it reaches $3 \pi / 8$, and, after this frequency, it decreases to $5 \pi / 8$ at $40 \mathrm{MHz}$.

\subsection{The Multi Layer Sensor}

To reduce the lateral resolution value, the magnetic field should be confined to a smaller spatial area by scaling the spiral, decreasing the width and pitch of the conductive tracks and 


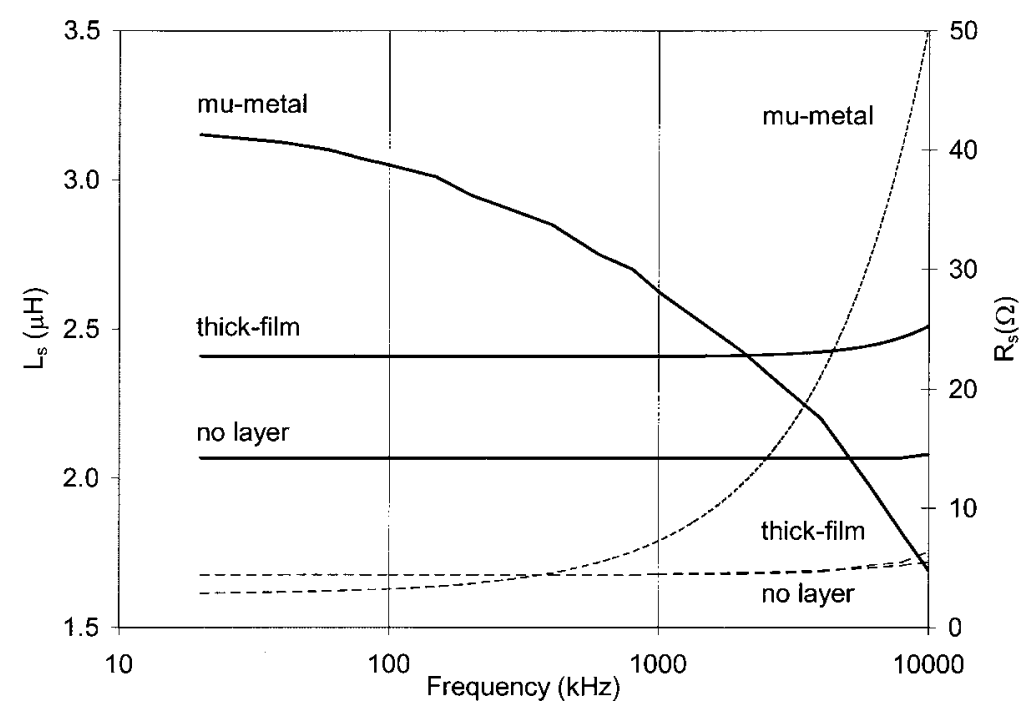

FIGURE $3 L_{S}$ (continuous lines) and $R_{S}$ (dot lines) as a function of frequency when material of different type and thickness are attached to the back of the sensor.

maintaining the same number of turns or more because the inductance value depends on the square of the number of turns and on the external diameter [10]. Since the screen pitch is near the low limit, the operation should be possible using a laser ablation technique or a gravure offset printing. This way, a track width down to a 50 and $35 \mu \mathrm{m}$ respectively $[11,12]$ can be reached; however, simpler way using a multi-layer structure is proposed. The sensor, schematically shown in Figure 4, consists of 10 overlapping spirals, each one separated by an insu-

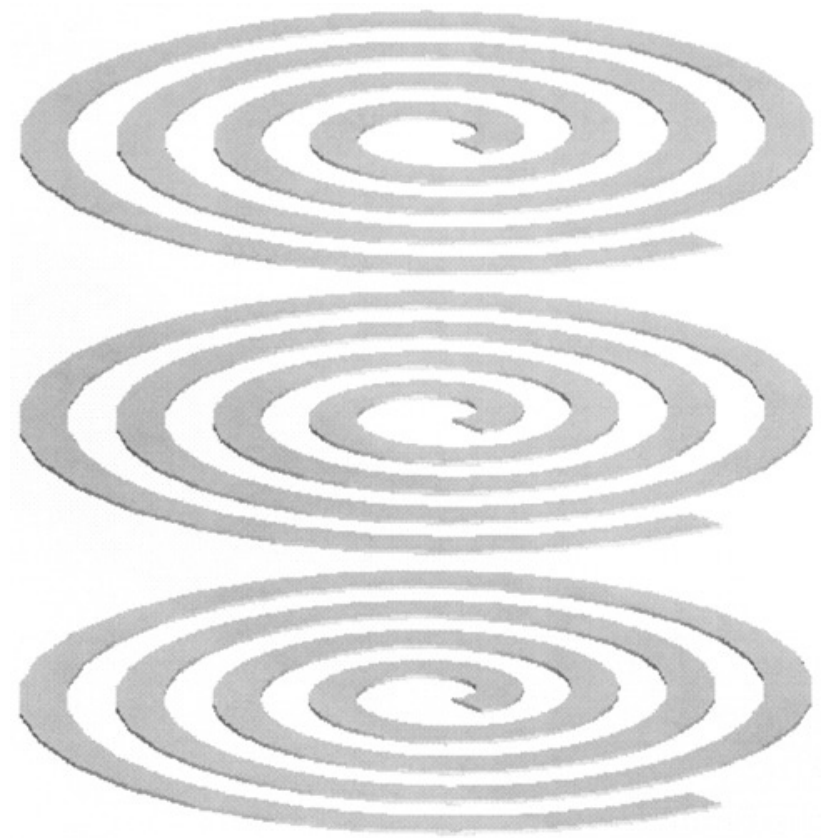

FIGURE 4 Schematic drawing of the multi layer sensor. 


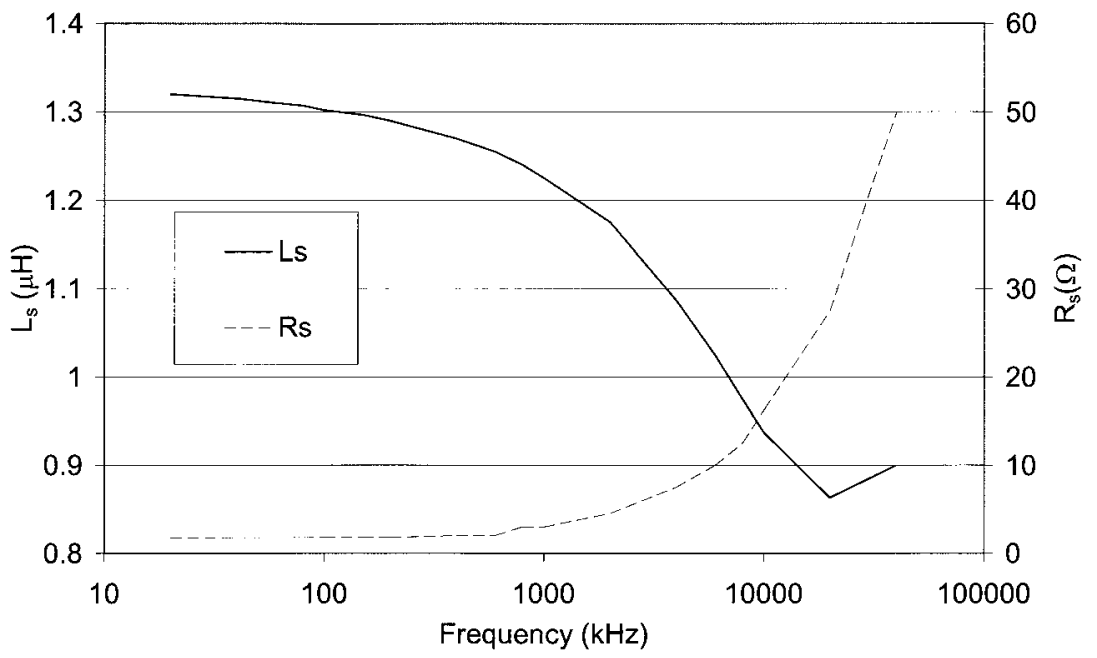

FIGURE 5 Equivalent series parameters of the multi layer sensor as a function of frequency.

lating layer; its thickness is about $400 \mu \mathrm{m}$. Each spiral has four turns, an external diameter of $3.6 \mathrm{~mm}$ and a $500 \mu \mathrm{m}$ pitch, while the width and the track thickness are respectively 250 and $20 \mu \mathrm{m}$. Each spiral is serially connected to increase the $L_{s}$ value; in this case also $C_{p}$ increases but its final value remains neglectable. Looking at Figure 4, the bottom and the medium spirals are connected through their centers, while the medium and the top ones are connected through their external terminals. The sensor is screen printed on an alumina substrate of the same dimensions of the previous sensor. In this case, a mu-metal layer has also been attached to the back of the alumina substrate. The measured curves of the equivalent series parameters $L_{s}$ and $R_{s}$ are reported in Figure 5 as a function of frequency. The $L_{s}$ and $R_{s}$ values are $1.30 \mu \mathrm{H}$ and $1.78 \Omega$ at $150 \mathrm{kHz}$, while $C_{p}$ is again negligible because the corresponding impedance phase rises toward $\pi / 2$ up to units of $\mathrm{MHz}$ where it begins to decrease reaching $3 \pi / 8$ at $40 \mathrm{MHz}$. Figure 6 shows the pictures of the two sensors: in (a) the single layer is reported; the dark zone corresponds to the spiral covered by the dielectric films and the two terminals are visible, in (b) front and side views of the multi-layer are shown. In the side view, it is possible to compare the height of the sensor with the solderings on the left.

\section{SENSITIVITY AND TEMPERATURE CROSS-SENSITIVITY}

A detailed study on the sensitivity should consider the relation between the measured quantities $R_{s}$ and $L_{s}$ and the physical effects, that is, the eddy current and the reluctance change. Since a mathematical determination of this relation is quite a complicated operation, an experimental approach is more suitable.

From the experimental point of view, the sensitivity depends mainly on (i) the material of the target, (ii) the working frequency and (iii) the measurement range. Because the materials can be classified into ferromagnetic and non-ferromagnetic, two different targets, one of iron and the other of brass have been prepared. Each target has been moved away from the sensor by a step of $200 \mu \mathrm{m}$ in the axial direction and the variations induced $(\Delta L$ and $\Delta R$ ) on the multi layers sensor have been measured as a function of frequency. Their values are reported in Figure 7(a) and (b). As the frequency rises, $\Delta L$ decreases and $\Delta R$ increases for both materials due to the eddy current losses and skin effect. The figure shows that, once the material 


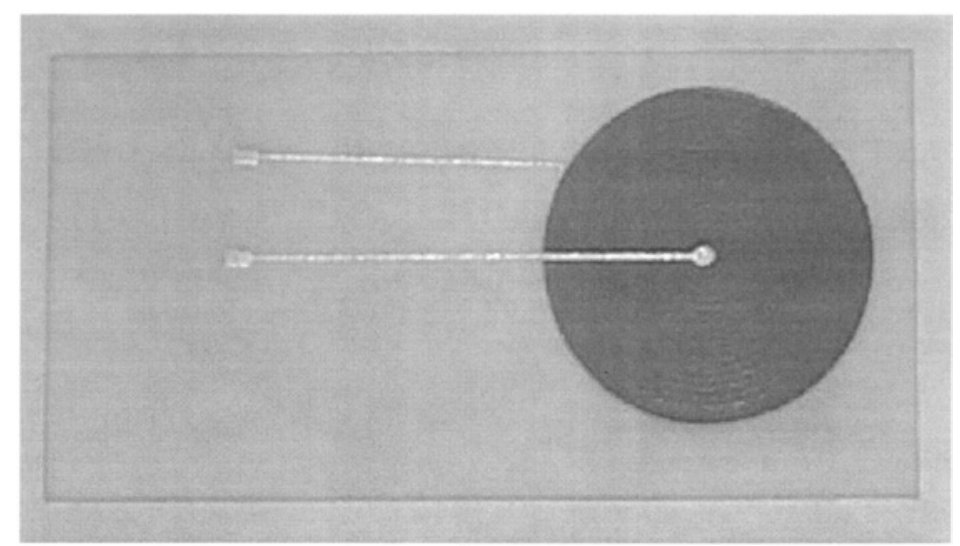

(a)

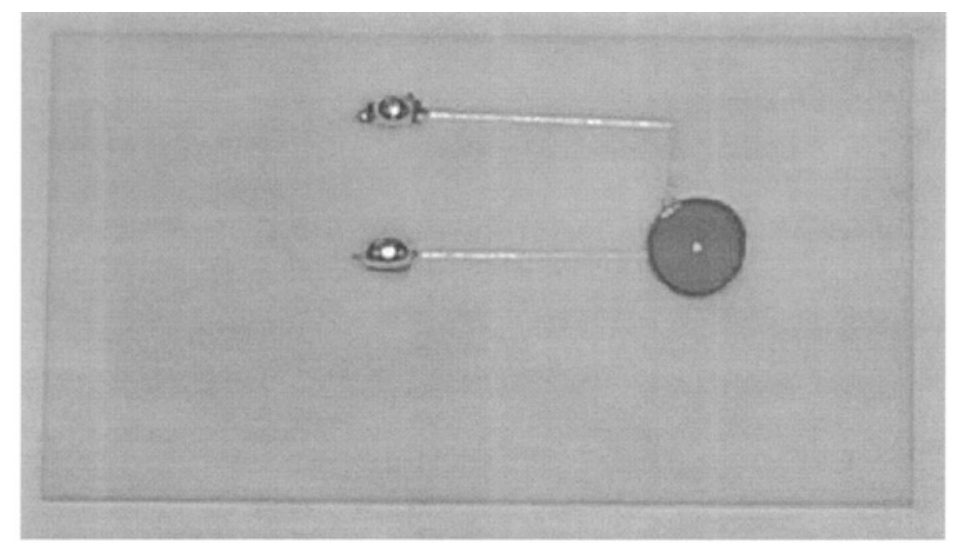

(b)

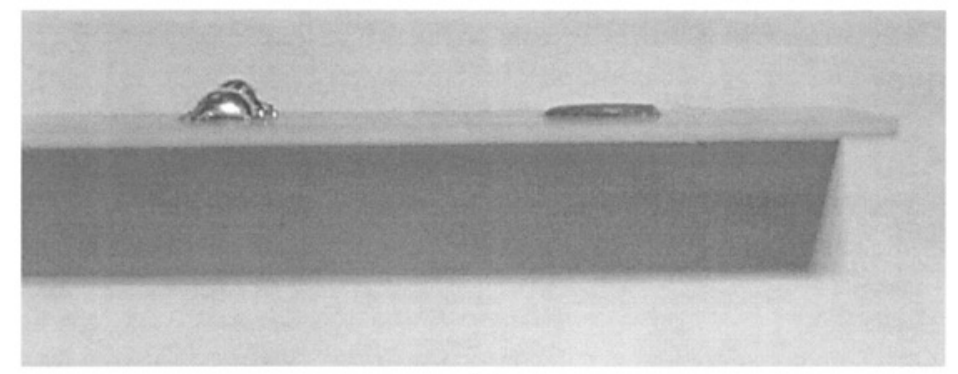

(c)

FIGURE 6 Pictures of the (a) single and (b) multi-layers planar sensors: the two swellings on the left are the solderings.

used in the specific application is known, optimized values of the sensitivity depend on an appropriate choice of the working frequency. When the specific application is defined, because $\Delta L$ and $\Delta R$ values change, the choice of the working frequency optimizes the sensitivity. Moreover, because the $\Delta L$ sign depends on the material type, the nature of the target can be recognized.

The sensitivity is a non-linear function of the distance. Simulation results suggest that the sensitivity has a maximum at a distance of about 200 microns from the sensor. To evaluate the cross sensitivity to temperature, the $L_{S}$ and $R_{S}$ values have been measured as a function of 


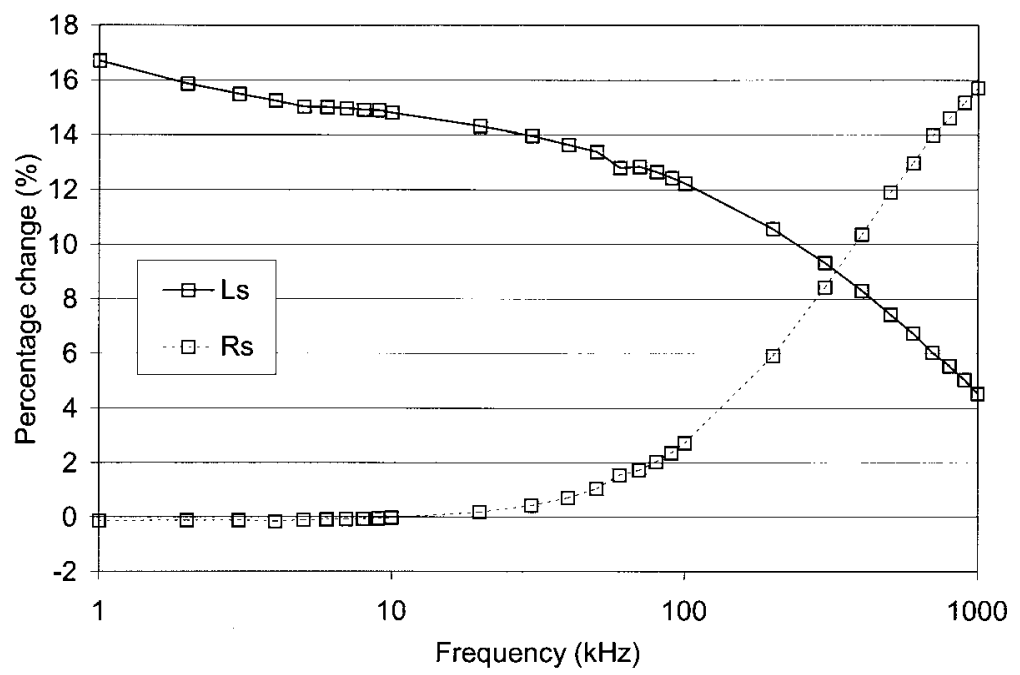

(a)

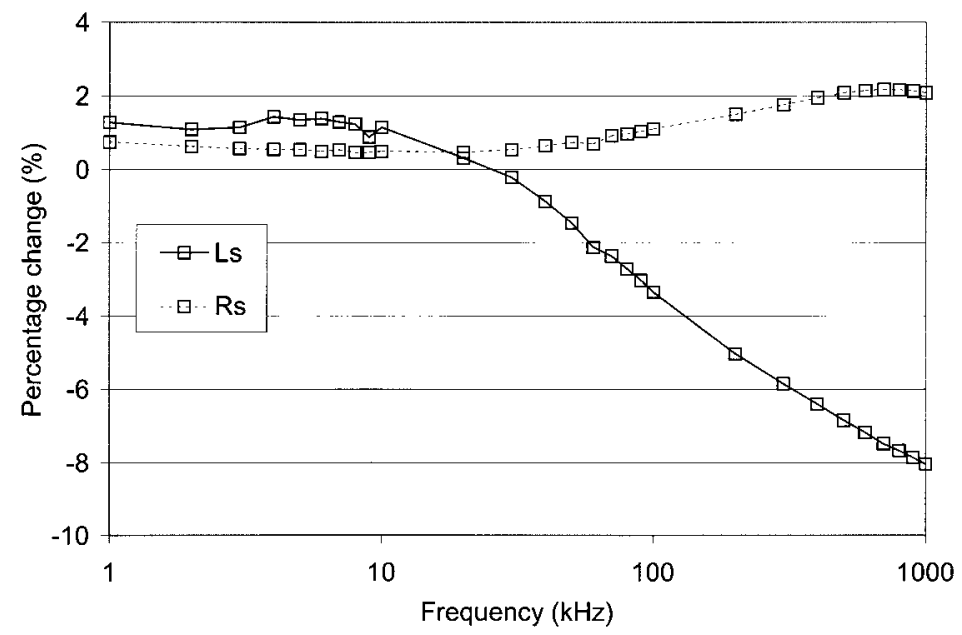

(b)

FIGURE 7 Percentage change of $R_{s}$ and $L_{s}$ due to (a) a ferromagnetic (iron) target and (b) non-ferromagnetic (brass) target when moved for a $200 \mu \mathrm{m}$ step.

temperature with the set-up shown in Figure 8(a). On the upper face of the alumina substrate the multi-layer sensor and the temperature sensor (Pt100) are visible, while on the lower face there is the heater. The $L_{S}$ and $R_{S}$ curves, evaluated at $150 \mathrm{kHz}$, are shown as a function of temperature in Figure 8(b): the inductance changes much less than the resistance, and is in the order of $50 \mathrm{ppm} /{ }^{\circ} \mathrm{C}$. These results agree with the theory because the inductance change is caused mainly by a geometric variation of the conductive tracks, while the resistance is strongly influenced by the resistivity dependance on temperature of the conductive paste resistivity.

\section{CONDITIONING AND MEASURING ELECTRONICS}

The measurement circuit is based on a synchronous demodulation technique and measures the $\Delta R$ and $\Delta L$ quantities separately. The working frequency has been chosen at $150 \mathrm{kHz}$ 


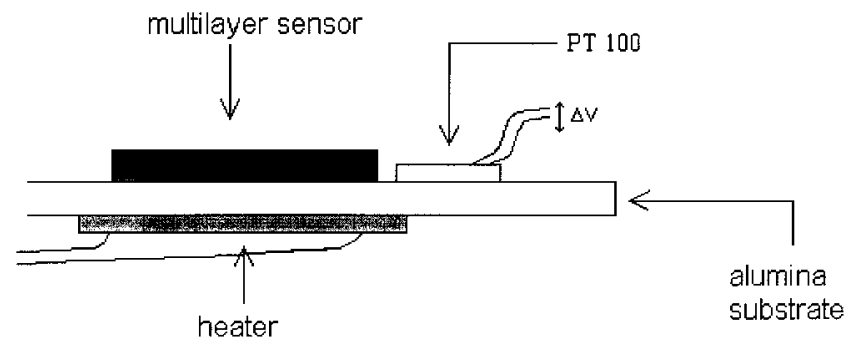

(a)

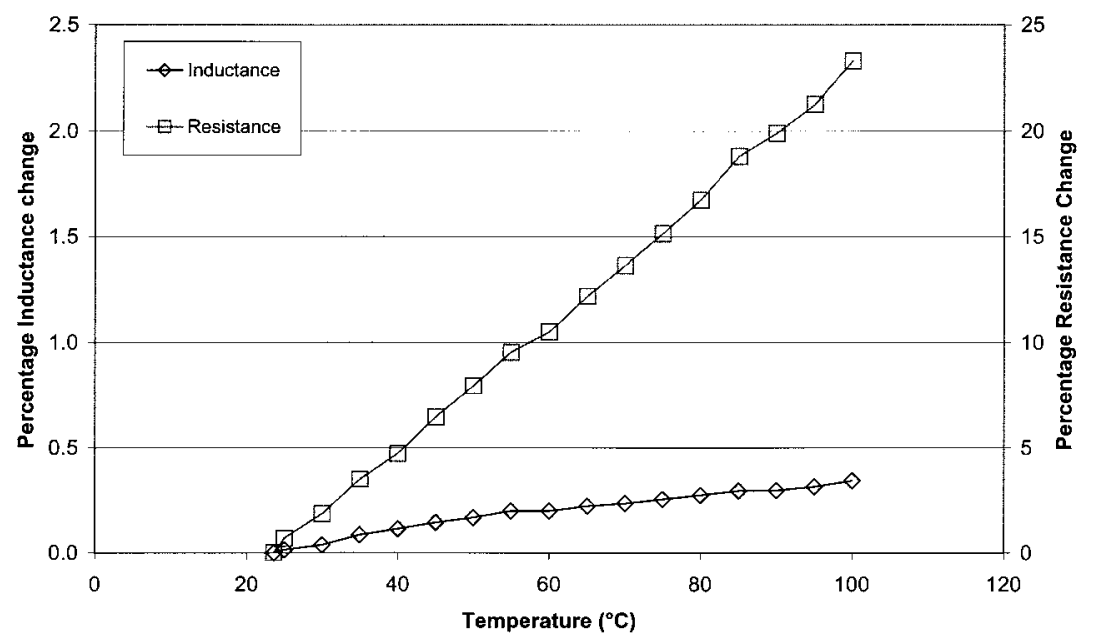

(b)

FIGURE 8 In (a) set-up for the sensibility to temperature measurement, in (b) $L_{s}$ and $R_{s}$ parameters as functions of temperature.

because at that frequency the $\Delta L$ values are a good compromise for both types of materials. Figure 9 shows a block scheme of the circuit. $V_{s}$ is a sinusoidal voltage at $150 \mathrm{kHz}$ frequency that supplies two voltages to current converters generating each an $I$ current of the same amplitude and frequency but opposite in phase. The reference is a series of an inductor and a resistor of the same value $L$ and $R$ of the sensor in a known condition, while $\Delta L$ and $\Delta R$ represent the variation induced by the changing of the quantity under measurement. After summing the voltages of the sensor and the reference caused by the $I$ current, $V_{\text {diff }}$ is equal to:

$$
V_{\mathrm{diff}}=k V_{s}(j \omega \Delta L+\Delta R)
$$

In the time domain, supposing $V_{s}$ equal to $\cos (\omega t), V_{\text {diff }}$ becomes:

$$
V_{\text {diff }}=k[\Delta R \cos (\omega t)+\omega \Delta L \sin (\omega t)]
$$

$V_{\text {diff }}$ then multiplies two signals equal to $V_{s}$ but the second is leading $\pi / 2$ with respect to the same $V_{s}$. The corresponding outputs, after the low pass filters are equal to:

$$
\begin{aligned}
V_{\Delta R} & =\frac{1}{2} k \Delta R \\
V_{L L} & =-k_{L} \mathbf{\square}_{L} L .
\end{aligned}
$$




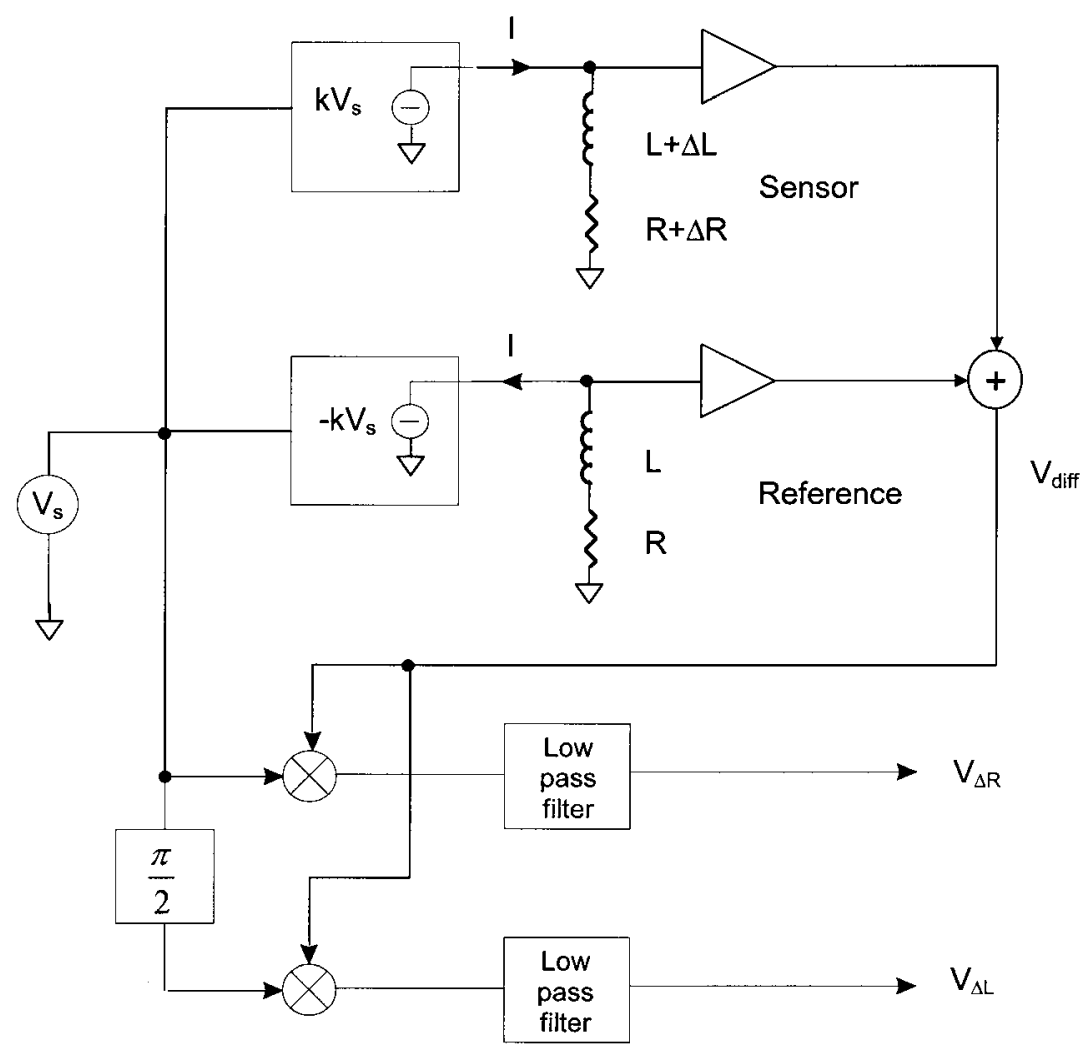

FIGURE 9 Block scheme of the conditioning electronics.

The cut-off frequencies of the two low pass filters are both located at $10 \mathrm{~Hz}$.

$$
V_{\Delta L}=\frac{1}{2} k \omega \Delta L
$$

The two sensors have been tested in the laboratory, the single layer applied as distance measurement, while the second as profile measurements.

\subsection{Distance Measurement}

A target, whose material is iron, with a cylindrical shape of $30 \mathrm{~mm}$ diameter is moved perpendicular to the sensor in steps of $100 \mu \mathrm{m}$ amplitude. Figure 10(a) reports the $V_{\Delta L}$ and $V_{\Delta R}$ output voltages as a function of the distance of the target from the sensor. A sensitivity curve, obtained by subtracting the output voltages of two adjacent experimental data and dividing the difference with the distance between these two points, ranges from a maximum of $-14 \mathrm{mV} / \mu \mathrm{m}$, at about $200 \mu \mathrm{m}$ distance down to $-2 \mathrm{mV} / \mu \mathrm{m}$.

Moreover, in order to calculate the resolution, the electronic noise has been measured and its standard deviation is $\sigma=8.60 \mathrm{mV}$ and $\sigma=10.34 \mathrm{mV}$ for $V_{\Delta L}$ and $V_{\Delta R}$ outputs respectively corresponding to a resolution of $0.6 \mu \mathrm{m}$ and $1.3 \mu \mathrm{m}$. 


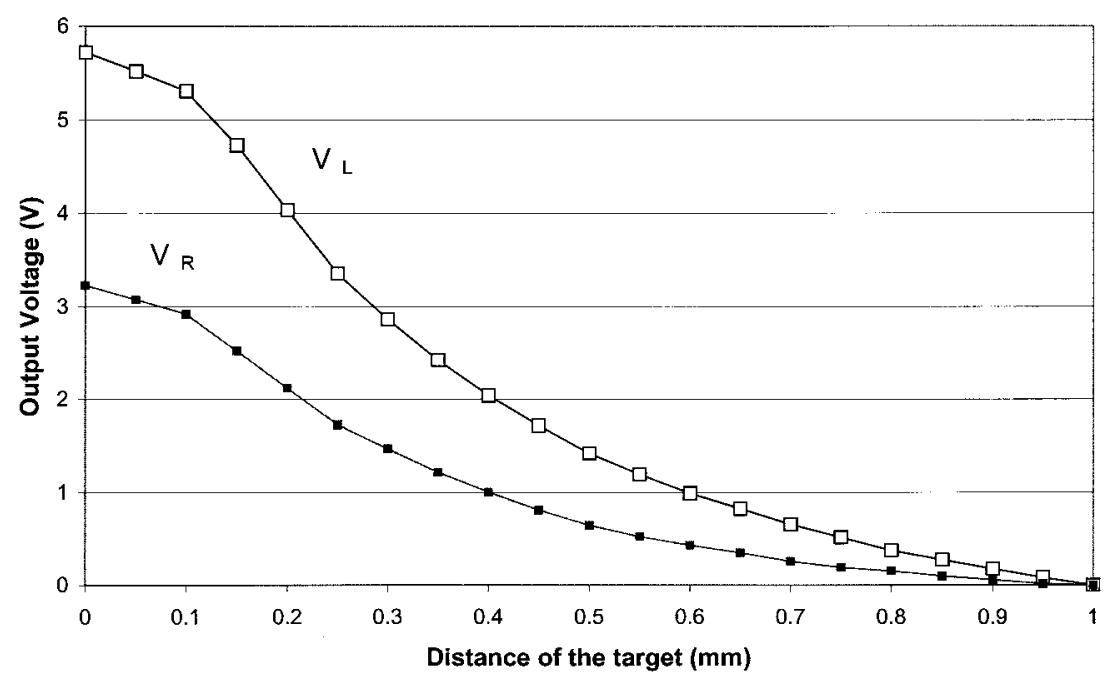

FIGURE 10 Output voltages of a single layer sensor as a function of the distance of a $30 \mathrm{~mm}$ diameter target from the sensor.

\subsection{Profile Reconstruction}

A metallic object, whose surface has two steps having amplitudes of tens of $\mu \mathrm{m}$, has been used to test the multi layer sensor. The profile of the object has been previously determined by using an optical instrument with a resolution of $0.2 \mu \mathrm{m}$, and a movement step in the lateral direction of $50 \mu \mathrm{m}$. The measurements obtained are reported as a continuous line in Figure 11. As it can be seen the heights of the steps are 50 and $30 \mu \mathrm{m}$ approximately.

The higher surface of the metallic object has been placed at about $200 \mu \mathrm{m}$ from the sensor and the object has been moved in the lateral direction of equal steps of $200 \mu \mathrm{m}$. The $V_{\Delta L}$ measured are reported as square data and connected by a dot line in Figure 11(a). Far from the

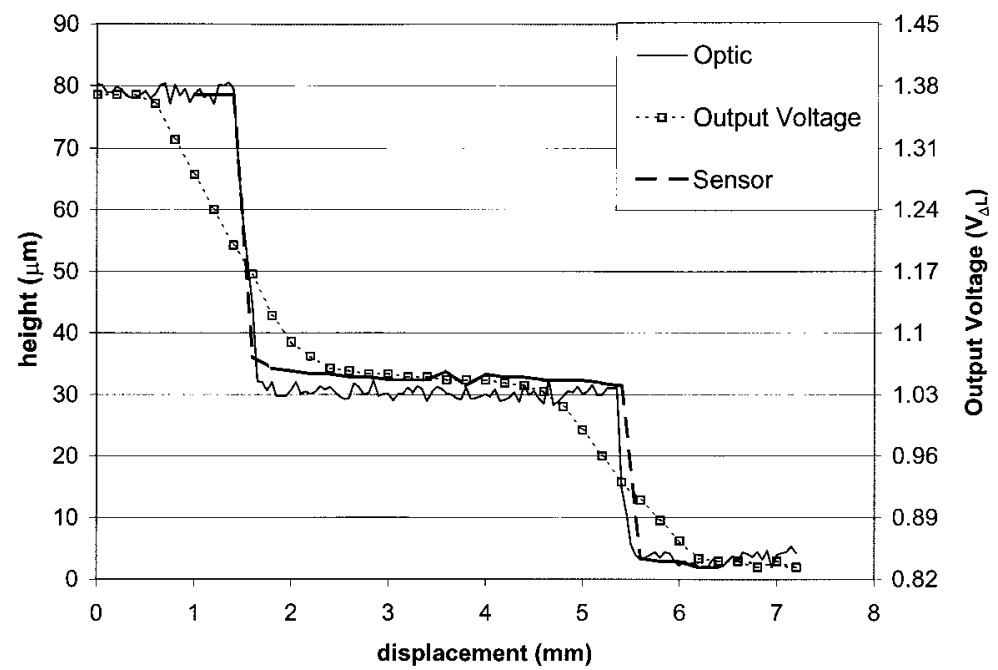

FIGURE 11 In a continuous line, the profile of a metallic object as measured by an optical profilometer. The dot line represents the output voltage due to an inductance change as measured by the sensor. 
edges, the two curves agree quite well being the difference less than few micrometers. Close to the edges, because the magnetic field is divided between the two planes, the $V_{\Delta L}$ measurements reconstructs a line that is not as sharp as the real profile. When the sensor is exactly over the edge, the magnetic field is divided into approximately equal parts, and the value measured is half the maximum of the height of the step. The $V_{\Delta L}$ data are modified according to the following algorithm:

(i) computation for each measured point of average on the preceding and following four points (equivalent to the averaging over $2 \mathrm{~mm}$ );

(ii) if the average is less than the value of the point, assign to the point the maximum value of the data used to calculate the average;

(iii) if the average is greater than the value of the point, assign to the point the minimum value of the data used to calculate the average.

Applying this algorithm, a line (thick and dotted) is reported in Figure 11, showing that the lateral resolution is corrected to less than $200 \mu \mathrm{m}$.

Although $V_{\Delta R}$ is available, only the $V_{\Delta L}$ has been reported because it has more resolution, and is much less sensitive to temperature; thus doesn't require a temperature compensation. In any case, the shape of the $V_{\Delta R}$ output voltage strictly looks like its $V_{\Delta L}$ output voltage.

\section{CONCLUSIONS}

Two planar inductive sensors proposed for distance and profile measurement have been developed by thick film technology since it offers the possibility to have a low cost/ performance ratio in presence of low and medium volume production. The first one is a spiral-shaped single layer while the second one is a multi-layered structure consisting of ten spirals one over the other. Simulation results demonstrate that a more concentrated magnetic field is generated when the internal terminal of the spiral starts from its center. An analysis of the sensitivity shows that the optimized value depends on the choice of the working frequency. Both sensors have been shielded from interference out of the sensitive area, by attaching a mu-metal layer to the back of the sensor support. Their cross sensitivity to temperature has been measured showing that the inductive and resistive components change by 50 and $2500 \mathrm{ppm} /{ }^{\circ} \mathrm{C}$ respectively. Laboratory tests show that the first sensor has a maximum sensitivity of $-14 \mathrm{mV} / \mu \mathrm{m}$, that combined with a standard deviation noise of $8.6 \mathrm{mV}$ gives a resolution of $0.6 \mu \mathrm{m}$, while the second one demonstrates a lateral resolution of $200 \mu \mathrm{m}$, after a software elaboration and an axial resolution of few $\mu \mathrm{m}$.

\section{References}

[1] Decker, W. and Kostka, P. (1989). Inductive and eddy current sensors. In: Göpel, W., Hesse, J. and Zemel, J. N. (Eds.), Sensors: A Comprehensive Survey, Vol. 5, Chap. 7. VCH, Weinheim, pp. 300-304.

[2] Passeraub, Ph. A., Besse, P. A., Hediger, S., de Raad, Ch. Dezuari, O., Quinet, F. and Popovic, R. S. (1998). Metallic profile and coin imaging using an inductive proximity sensor microsystem. Sensors and Actuators A, 66, 225-230.

[3] Bartoletti, C., Buonanni, R., Fantasia, L. G., Frulla, R., Gaggioli, W. and Sacerdoti, G. (1998). The design of a proximity inductive sensor. Meas. Sci. Technol., 9, 1180-1190.

[4] Passeraub, Ph. A., Besse, P. A., Hediger, S., de Raad, Ch. and Popovic, R. S. (1998). High resolution miniaturized inductive proximity sensor: Characterization and application for step-motor control. Sensors and Actuators A, 68, 257-262.

[5] Fenniri, H., Moineau, A. and Delaunay, G. (1994). Profile imagery using a flat eddy-current proximity sensor. Sensors and Actuators A, 45, 183-190. 
[6] Passeraub, Ph. A., Besse, P. A., de Raad, Ch. and Popovic, R. S. (1997). A differential relaxation oscillator as a versatile electronic interface for sensors. Sensor and Actuators A, 58, 141-148.

[7] Passeraub, Ph. A., Rey-Mermet, G., Besse, P. A., Lorenz, H. and Popovic, R. S. (1997). Inductive proximity sensor with a flat coil and a new differential relaxation oscillator. Sensor and Actuators A, 60, 122-126.

[8] Passeraub, Ph. A., Besse, P. A. and Popovic, R. S. (2000). Temperature compensation of an integrated low power inductive proximity microsensor. Sensors and Actuators A, 82, 62-68.

[9] Passeraub, Ph. A., Besse, P. A., Bayadroun, A., Hediger, S., Bernasconi, E., de Raad, Ch. and Popovic, R. S. (1999). First integrated proximity sensor with on-chip CMOS readout circuit and electrodeposited $1 \mathrm{~mm}$ flat coil. Sensors and Actuators A, 76, 273-278.

[10] Passeraub, Ph. A., Besse, P. A. and Popovic, R. S. (1996). Scaling down an inductive proximity sensor. Sensors and Actuators A, 52, 114-118.

[11] Kripesh, V., Bhatnagar, S. K., Osterwinter, H. and Gust, W. (1996). Fine-line passive components for hybrid microelectronics. Microelectronics International, 13, 9-11.

[12] Hagberg, J., Pudas, M., Leppavuori, S., Elsey, K. and Logan, A. (2001). Gravure offset printing development for fine line thick film circuits. Microelectronics International, 18(3), 32-35. 

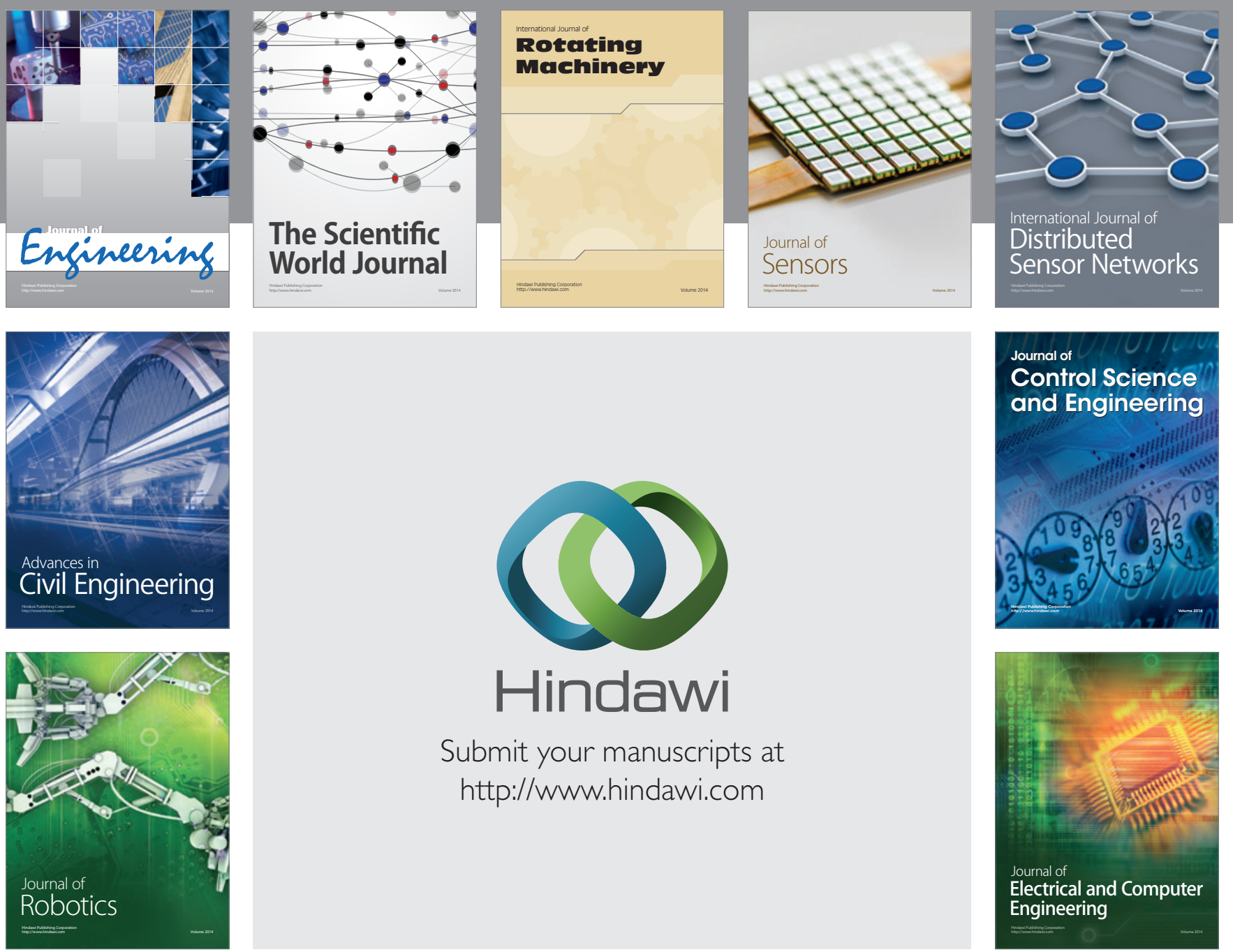

Submit your manuscripts at

http://www.hindawi.com
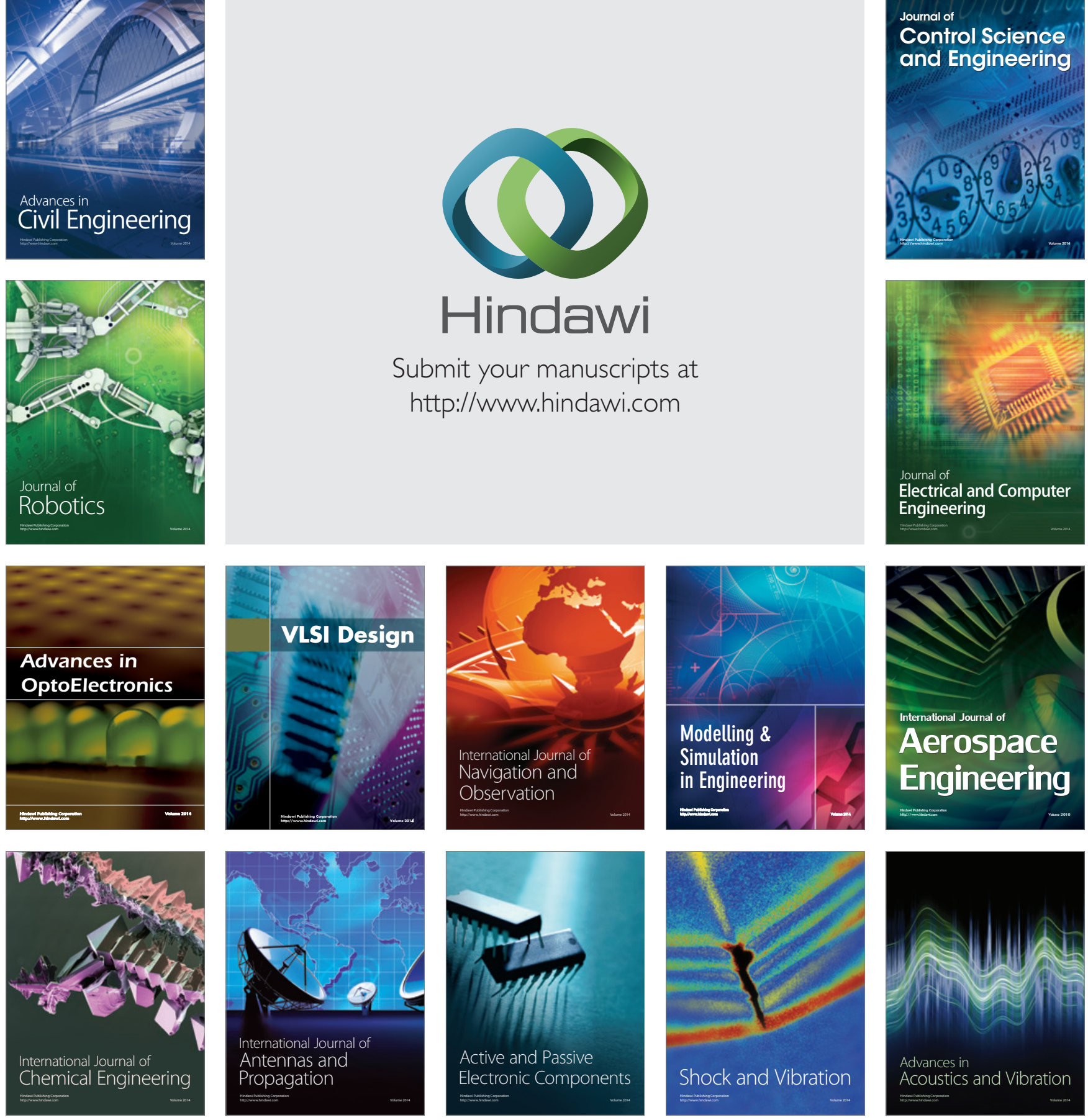